\title{
ON SPECTRAL PREDICTION ERROR FORMULAS FOR STATIONARY RANDOM FIELDS ON $Z^{2}$
}

\section{H. Niemi}

\section{Introduction}

We are concerned with analytical expressions for the prediction errors of second order stationary random fields $x_{m, n},(m, n) \in Z^{2}$. The study of prediction theory of stationary random fields goes back to Chiang Tse-Pei [1] and Helson and Lowdenslager [2], [3]. More recently several authors have treated different kinds of prediction theoretical problems for stationary random fields, cf. e.g. [4]-[11] and [13].

Let $x_{m, n},(m, n) \in Z^{2}$, be a stationary random field. Mainly the following prediction problems have been treated in literature

(i) the half-plane prediction error

$$
\left\|e^{1}(x)\right\|^{2}=\left\|x_{0,0}-\operatorname{Proj}_{\overline{s p}\left\{x_{j, k}: j<0, k \in Z\right\}} x_{0,0}\right\|^{2},
$$

(ii) the lexicographic prediction error

$$
\left\|e^{2}(x)\right\|^{2}=\left\|x_{0,0}-\operatorname{Proj}_{\overline{s p}\left\{x_{j, k}: j<0, k \in Z \text { or } j=0, k<0\right\}} x_{0,0}\right\|^{2},
$$

(iii) the extended half-plane prediction error

$$
\left\|e^{3}(x)\right\|^{2}=\left\|x_{0,0}-\operatorname{Proj}_{\overline{s p}\left\{x_{j, k}: j \leq 0, k \in Z,(j, k) \neq(0,0)\right\}} x_{0,0}\right\|^{2},
$$

(iv) the quarter-plane prediction error

$$
\left\|e^{4}(x)\right\|^{2}=\left\|x_{0,0}-\operatorname{Proj}_{\overline{s p}\left\{x_{j, k}: j<0, k<0\right\}} x_{0,0}\right\|^{2} .
$$

Analytical expressions for $\left\|e^{1}(x)\right\|^{2}$ were obtained independently in [5] and [8] (cf. [1]) and, respectively, for $\left\|e^{2}(x)\right\|^{2}$ in [2]. Corresponding results for $\left\|e^{3}(x)\right\|^{2}$ have been obtained in [6] and [11].

Our main result is an analytical expression for $\left\|e^{4}(x)\right\|^{2}$ under the strong commutation condition, introduced in [4] (cf. Theorem 3.9 and 3.10). Our results are based on the four-fold Wold decomposition for stationary random fields having the strong commutation property obtained by Kallianpur and Mandrekar [4] and its spectral counterpart obtained by Korezlioglu and Loubaton [8] (cf. [5]). We 
also make use of the spectral representation theorems for the horizontal and, respectively, vertical innovation fields of $x_{m, n},(m, n) \in Z^{2}$, obtained by Korezlioglu and Loubaton [8].

As noted earlier, our main results are derived under the strong commutation condition. Sufficient spectral conditions for the strong commutation condition to hold have been obtained by Soltani [13] and Miamee and Niemi [10].

\section{Geometrical interpretation}

Let $\left\{x_{m, n}\right\}$ be a stationary random field. The information sets generated by observations $x_{m, n},(m, n) \in \mathcal{S}\left(\subset Z^{2}\right)$, are defined as closed linear subspaces of $L^{2}(\Omega, \mathcal{A}, P)$ as follows:

$$
\begin{aligned}
& H_{x}=\overline{s p}\left\{x_{j, k}:(j, k) \in Z^{2}\right\}, \\
& H_{x}^{1}(m)=\overline{s p}\left\{x_{j, k}: j \leq m, k \in Z\right\}, \quad H_{x}^{1}(-\infty)=\bigcap_{m \in Z} H_{x}^{1}(m), \\
& H_{x}^{2}(n)=\overline{s p}\left\{x_{j, k}: j \in Z, k \leq n\right\}, \quad H_{x}^{2}(-\infty)=\bigcap_{n \in Z} H_{x}^{2}(n), \\
& H_{x}^{1+}(m, n)=H_{x}^{1}(m) \bigvee \overline{s p}\left\{x_{m+1, k}: k \leq n\right\}, \\
& H_{x}^{2+}(m, n)=H_{x}^{2}(n) \bigvee \overline{s p}\left\{x_{j, n+1}: j \leq m\right\}, \\
& H_{x}^{*}(m, n)=\overline{s p}\left\{x_{j, k}: j \leq m \text { or } k \leq n\right\}, H_{x}(m, n)=\overline{s p}\left\{x_{j, k}: j \leq m, k \leq n\right\}
\end{aligned}
$$

and, in general, for an arbitrary $\mathcal{S} \subset Z^{2}$

$$
H_{x}(\mathcal{S})=\overline{s p}\left\{x_{j, k}:(j, k) \in \mathcal{S}\right\}
$$

Furthermore, for any closed linear subspace $M \subset H_{x}$ we define

$$
x_{m, n} / M=\operatorname{Proj}_{M} x_{m, n}, \quad(m, n) \in Z^{2} .
$$

2.1. Definition. A stationary random field $\left\{x_{m, n}\right\}$ is
(a) horizontally deterministic, if $H_{x}^{1}(-\infty)=H_{x}$,
(b) horizontally purely non-deterministic, if $H_{x}^{1}(-\infty)=\{0\}$,
(c) vertically deterministic, if $H_{x}^{2}(-\infty)=H_{x}$,
(d) vertically purely non-deterministic, if $H_{x}^{2}(-\infty)=\{0\}$,
(e) strongly purely non-deterministic, if $H_{x}^{1}(-\infty)=H_{x}^{2}(-\infty)=\{0\}$. 
Recall that any stationary random field $\left\{x_{m, n}\right\}$ admits two-fold Wold decompositions of the form

$$
x_{m, n}=R_{m, n}^{i}(x)+S_{m, n}^{i}(x),
$$

with

$$
S_{m, n}^{i}(x)=x_{m, n} / H_{x}^{i}(-\infty), \quad R_{m, n}^{i}(x)=x_{m, n}-S_{m, n}^{i}(x), \quad i=1,2 .
$$

The component $\left\{R_{m, n}^{1}(x)\right\}$ (respectively $\left\{R_{m, n}^{2}(x)\right\}$ ) is horizontally (respectively vertically) purely non-deterministic and $\left\{S_{m, n}^{1, n}(x)\right\}$ (respectively $\left\{S_{m, n}^{2}(x)\right\}$ ) is horizontally (respectively vertically) deterministic. The stationary random fields

$$
W_{m, n}^{1}(x)=x_{m, n}-x_{m, n} / H_{x}^{1}(m-1)
$$

respectively

$$
W_{m, n}^{2}(x)=x_{m, n}-x_{m, n} / H_{x}^{2}(n-1)
$$

are the horizontal (respectively vertical) innovations of $\left\{x_{m, n}\right\}$. It is well-known that

$$
H_{R^{i}(x)}^{i}(m)=H_{W^{i}(x)}^{i}(m), \quad i=1,2 .
$$

2.3. Remark. Our method to obtain an analytical expression for the prediction error (1.4) is based on the spectral representation of the innovation fields $\left\{W_{m, n}^{i}(x)\right\}, i=1,2$. The formula (2.4), going back to Korezlioglu and Loubaton [8], p. 155 , shows that $e^{2}(x)$ can be obtained as the one-step prediction error of the stationary sequence $\left\{W_{0, n}^{1}\right\}_{n \in Z}$.

2.4. Proposition. Let $\left\{x_{m, n}\right\}$ be a stationary random field. Then

$$
e^{2}(x)=W_{0,0}^{1}(x)-W_{0,0}^{1}(x) / H_{W^{1}(x)}\left(\left\{(j, k) \in Z^{2}: j=0, k<0\right\}\right) .
$$

The next commutativity property was introduced by Kallianpur and Mandrekar [4].

2.5. Definition. A stationary random field $\left\{x_{m, n}\right\}$ has the strong commutation property, if

$$
\operatorname{Proj}_{H_{x}^{1}(m)} \operatorname{Proj}_{H_{x}^{2}(n)}=\operatorname{Proj}_{H_{x}(m, n)}, \quad(m, n) \in Z^{2} .
$$

2.6. Remark. Each of the conditions (2.6.a) and (2.6.b) is equivalent to the strong commutation property:

$$
\begin{aligned}
& \operatorname{Proj}_{H_{x}^{1}(m)} \operatorname{Proj}_{H_{x}^{2}(n)}=\operatorname{Proj}_{H_{x}^{2}(n)} \operatorname{Proj}_{H_{x}^{1}(m)} \text { and } \\
& H_{x}(m, n)=H_{x}^{1}(m) \cap H_{x}^{2}(n), \quad(m, n) \in Z^{2}, \\
& H_{x}^{1}(m) \ominus H_{x}(m, n) \perp H_{x}^{2}(n) \ominus H_{x}(n, n), \quad(m, n) \in Z^{2} .
\end{aligned}
$$


2.7. Lemma. Let $\left\{x_{m, n}\right\}$ be a stationary random field having the strong commutation property. Then

$$
\begin{aligned}
& H_{x}^{*}(m, n)=H_{x}(m, n) \oplus\left[H_{x}^{1}(m) \ominus H_{x}(m, n)\right] \oplus\left[H_{x}^{2}(n) \ominus H_{x}(m, n)\right], \\
& \text { (2.7.b) } H_{x}\left(\left\{(j, k) \in Z^{2}: j \leq m, k \leq n\right\} \backslash\{(m, n)\}\right)= \\
& H_{x}(m-1, n-1) \oplus\left[H_{x}(m, n-1) \ominus H_{x}(m-1, n-1)\right] \\
& \oplus\left[H_{x}(m-1, n) \ominus H_{x}(m-1, n-1)\right], \\
& \text { (2.7.c) } H_{x}^{1+}(m, n)=H_{x}(m, n) \oplus\left[H_{x}^{1}(m) \ominus H_{x}(m, n)\right] \\
& \oplus\left[H_{x}(m+1, n) \ominus H_{x}(m, n)\right] \text {, } \\
& \text { (2.7.d) } H_{x}^{2+}(m, n)=H_{x}(m, n) \oplus\left[H_{x}^{2}(n) \ominus H_{x}(m, n)\right] \\
& \oplus\left[H_{x}(m, n+1) \ominus H_{x}(m, n)\right] \text {, } \\
& \text { (2.7.e) } \quad H_{x}(m, n) \ominus H_{x}(m, n-1)=H_{W^{2}(x)}\left(\left\{(j, k) \in Z^{2}: j \leq m, k=n\right\}\right) \text {, } \\
& \text { (2.7.f) } \quad H_{x}(m, n) \ominus H_{x}(m-1, n)=H_{W^{1}(x)}\left(\left\{(j, k) \in Z^{2}: j=m, k \leq n\right\}\right) \text {. }
\end{aligned}
$$

Proof. The statements (2.7.a-d) are obvious. By symmetry it is enough to present a proof only to the first one of the statements (2.7.e-f). Denote,

$$
\mathcal{S}(m-, n)=\left\{(j, k) \in Z^{2}: j \leq m, k=n\right\},
$$

and

$$
M=\left\{z-\operatorname{Proj}_{H_{x}^{2}(n-1)} z: z \in H_{x}(\mathcal{S}(m-, n))\right\} .
$$

It is obvious that $M=H_{W^{2}(x)}(\mathcal{S}(m-, n))$. Moreover, by the strong commutativity

$$
\begin{aligned}
\operatorname{Proj}_{H_{x}^{2}(n-1)} z & =\operatorname{Proj}_{H_{x}^{2}(n-1)} \operatorname{Proj}_{H^{1}(m)} z \\
& =\operatorname{Proj}_{H_{x}(m, n-1)} z, \quad z \in H_{x}(\mathcal{S}(m-, n)),
\end{aligned}
$$

showing that

$$
M=\left\{z-\operatorname{Proj}_{H_{x}(m, n-1)} z: z \in H_{x}(\mathcal{S}(m-, n))\right\} .
$$

Since for all $z \in H_{x}(m, n-1), z-\operatorname{Proj}_{H_{x}(m, n-1)} z=0$, it is then obvious that

$$
M=\left\{z-\operatorname{Proj}_{H_{x}(m, n-1)} z: z \in H_{x}(m, n)\right\}=H_{x}(m, n) \ominus H_{x}(m, n-1) .
$$

The next result shows that the *-prediction problem, introduced in [13], reduces to the lexicographical one when $\left\{x_{m, n}\right\}$ has the strong commutation property. The fact that

$$
x_{m, n} / H_{x}^{1+}(m-1, n-1)=x_{m, n} / H_{x}^{2+}(m-1, n-1), \quad(m, n) \in Z^{2},
$$


for any stationary random field $\left\{x_{m, n}\right\}$ having the strong commutation property has been proved already by Korezlioglu and Loubaton [7; Proposition 2.1.4] (under the assumption $\left.x_{m, n} / H_{x}^{1+}(m-1, n-1) \neq 0\right)$.

2.8. Proposition. Let $\left\{x_{m, n}\right\}$ be a stationary random field having the strong commutation property. Then

$$
\begin{aligned}
& x_{m, n} / H_{x}^{*}(m-1, n-1)=x_{m, n} / H_{x}^{1+}(m-1, n-1) \\
& =x_{m, n} / H_{x}^{2+}(m-1, n-1) \\
& =x_{m, n} / H_{x}\left(\left\{(j, k) \in Z^{2}: j \leq m, k \leq n\right\} \backslash\{(m, n)\}\right) .
\end{aligned}
$$

Proof. By Lemma 2.7

$$
\begin{aligned}
x_{m, n} / H_{x}^{*}(m-1, n-1) & =\operatorname{Proj}_{H_{x}(m-1, n-1)} x_{m, n} \\
& +\left[\operatorname{Proj}_{H_{x}^{1}(m-1)}-\operatorname{Proj}_{H_{x}(m-1, n-1)}\right] x_{m, n} \\
& +\left[\operatorname{Proj}_{H_{x}^{2}(n-1)}-\operatorname{Proj}_{H_{x}(m-1, n-1)}\right] x_{m, n}
\end{aligned}
$$

Furthermore, by the strong commutativity

$$
\operatorname{Proj}_{H_{x}^{1}(m-1)} x_{m, n}=\operatorname{Proj}_{H_{x}^{1}(m-1)} \operatorname{Proj}_{H_{x}^{2}(n)} x_{m, n}=\operatorname{Proj}_{H_{x}(m-1, n)} x_{m, n}
$$

and by symmetry

$$
\operatorname{Proj}_{H_{x}^{2}(n-1)} x_{m, n}=\operatorname{Proj}_{H_{x}(m, n-1)} x_{m, n},
$$

yielding together with (2.7.b-d) all the equalities in (2.9).

In what follows we make heavy use of the four-fold Wold decompositon theorem obtained by Kallianpur and Mandrekar [4]. According to Theorems 2.1 and 2.2 in [4] any stationary random field having the strong commutation property admits a representation of the form

$$
x_{m, n}=\xi_{m, n}+\zeta_{m, n}^{1}+\zeta_{m, n}^{2}+\eta_{m, n}, \quad(m, n) \in Z^{2}
$$

where all the components are mutually orthogonal stationary random fields having the strong commutation property and

$$
\begin{aligned}
H_{x}(m, n)=H_{\xi}(m, n) & \oplus H_{\zeta^{1}}(m, n) \\
& \oplus H_{\zeta^{2}}(m, n) \oplus H_{\eta}(m, n), \quad(m, n) \in Z^{2} .
\end{aligned}
$$

Moreover,

$$
\begin{cases}H_{\xi}^{1}(-\infty)=H_{\xi}^{2}(-\infty)=\{0\}, & H_{\zeta^{1}}^{1}(-\infty)=H_{\zeta^{2}}^{2}(-\infty)=\{0\} \\ H_{\zeta^{1}}^{2}(-\infty)=H_{\zeta^{1}}, & H_{\zeta^{2}}^{1}(-\infty)=H_{\zeta^{2}} \\ H_{\eta}^{1}(-\infty)=H_{\eta}^{2}(-\infty)=H_{\eta} & \end{cases}
$$


2.11. Theorem. Let $\left\{x_{m, n}\right\}$ be a stationary random field having the strong commutation property. If $\left\{x_{m, n}\right\}$ is strongly purely non-deterministic, then

$$
e^{4}(x)=W_{0,0}^{1}(x)+W_{0,0}^{2}(x)-d_{0,0}(x)
$$

with

$$
d_{0,0}(x)=x_{0,0}-x_{0,0} / H_{x}^{1+}(-1,-1)=x_{0,0}-x_{0,0} / H_{x}^{2+}(-1,-1)
$$

and

$$
\left\|e^{4}(x)\right\|^{2}=\left\|W_{0,0}^{1}(x)\right\|^{2}+\left\|W_{0,0}^{2}(x)\right\|^{2}-\left\|e^{2}(x)\right\|^{2}
$$

2.12. Theorem. Let $\left\{x_{m, n}\right\}$ be a stationary random field having the strong commutation property. Then

$$
\begin{aligned}
e^{4}(x)=\xi_{0,0}(x) & -\xi_{0,0}(x) / H_{\xi}(-1,-1)+\zeta_{0,0}^{1}(x)-\zeta_{0,0}^{1}(x) / H_{\zeta^{1}}^{1}(-1) \\
& +\zeta_{0,0}^{2}(x)-\zeta_{0,0}^{2}(x) / H_{\zeta^{2}}^{2}(-1)
\end{aligned}
$$

with

(2.12.b) $\xi_{0,0}-\xi_{0,0} / H_{\xi}(-1,-1)=W_{0,0}^{1}(\xi)+W_{0,0}^{2}(\xi)-d_{0,0}(\xi)$

and

$$
\begin{aligned}
& \left\|e^{4}(x)\right\|^{2}=\left\|W_{0,0}^{1}(\xi)\right\|^{2}+\left\|W_{0,0}^{2}(\xi)\right\|^{2}-\left\|e^{2}(\xi)\right\|^{2} \\
& \left.\quad+\left\|\zeta_{0,0}^{1}(x)-\zeta_{0,0}^{1}(x) / H_{\zeta^{1}}^{1}(-1)\right\|^{2}+\| \zeta_{0,0}^{2}(x)-\zeta_{0,0}^{2}(x) / H_{\zeta^{2}}^{2}(-1)\right) \|^{2}
\end{aligned}
$$

Proof of Theorem 2.11. We first notice that by the strong commutativity

$$
\begin{aligned}
x_{0,0}-x_{0,0} / H_{x}(-1,-1) & =\left(I-\operatorname{Proj}_{H_{x}^{1}(-1)} \operatorname{Proj}_{H_{x}^{2}(-1)}\right) x_{0,0} \\
& =\left(I-\operatorname{Proj}_{H_{x}^{2}(-1)} \operatorname{Proj}_{H_{x}^{1}(-1)}\right) x_{0,0} .
\end{aligned}
$$

Furthermore, for any projections $P_{1}$ and $P_{2}$ one has $I-P_{1} P_{2}=\left(I-P_{1}\right)+P_{1}(I-$ $P_{2}$ ). Thus, in the present case

$$
x_{0,0}-x_{0,0} / H_{x}(-1,-1)=W_{0,0}^{1}(x)+W_{0,0}^{2}(x) / H_{x}^{1}(-1) .
$$

Moreover, by the strong commutativity

$$
W_{0,0}^{2}(x) / H_{x}^{1}(-1)=\operatorname{Proj}_{H_{x}^{1}(-1)} \operatorname{Proj}_{H_{x}^{2}(0)} W_{0,0}^{2}(x)=\operatorname{Proj}_{H_{x}(-1,0)} W_{0,0}^{2}(x),
$$

and since $W_{0,0}^{2}(x) \perp H_{x}(-1,-1)$,

$$
\operatorname{Proj}_{H_{x}(-1,0)} W_{0,0}^{2}(x)=\operatorname{Proj}_{H_{x}(-1,0) \ominus H_{x}(-1,-1)} W_{0,0}^{2}(x) .
$$

By (2.7.e), with $\mathcal{S}((-1)-, 0)=\left\{(j, k) \in Z^{2}: j \leq-1, k=0\right\}$,

$$
H_{x}(-1,0) \ominus H_{x}(-1,-1)=H_{W^{2}(x)}(\mathcal{S}((-1)-, 0)) \text {. }
$$


This, together with Proposition 2.4, gives

$$
W_{0,0}^{2}(x) / H_{x}^{1}(-1)=W_{0,0}^{2}(x) / H_{W_{x}^{2}}(\mathcal{S}((-1)-, 0))=x_{0,0} / H_{x}^{1+}(-1,-1) .
$$

Thus, by applying (2.4) together with (2.9) we obtain

$$
\begin{aligned}
x_{0,0}-x_{0,0} / H_{x}^{1}(-1,-1) & =W_{0,0}^{1}(x)+W_{0,0}^{2}(x) \\
& -\left(W_{0,0}^{2}(x)-W_{0,0}^{2}(x)\right) / H_{W_{x}^{2}}(\mathcal{S}((-1)-, 0)) \\
& =W_{0,0}^{1}(x)+W_{0,0}^{2}(x)-d_{0,0}(x) .
\end{aligned}
$$

The proof of (2.11.b) is obvious.

Proof of Theorem 2.12. It clearly follows from the orthogonality property (2.10.b) of the four-fold decomposition (2.10.a) that

$$
\begin{aligned}
x_{0,0}-x_{0,0} / H_{x}(-1,-1) & =\xi_{0,0}-\xi_{0,0} / H_{\xi}(-1,-1)+\zeta_{0,0}^{1}-\zeta_{0,0}^{1} / H_{\zeta^{1}}(-1,-1) \\
& +\zeta_{0,0}^{2}-\zeta_{0,0}^{2} / H_{\zeta^{2}}(-1,-1)+\eta_{0,0}-\eta_{0,0} / H_{\eta}(-1,-1) .
\end{aligned}
$$

Since $H_{\eta}^{1}(-\infty)=H_{\eta}^{2}(-\infty)=H_{\eta}$ (cf. (2.10.c)) and since $\left\{\eta_{m, n}\right\}$ has the strong commutation property it is obvious that $H_{\eta}(-1,1)=H_{\eta}$, yielding

$$
\eta_{0,0}-\eta_{0,0} / H_{\eta}(-1,-1)=0
$$

Since $\left\{\zeta_{m, n}^{1}\right\}$ has the strong commutation property and is vertically deterministic,

$$
H_{\zeta^{1}}(-1,-1)=H_{\zeta^{1}}^{1}(-1)
$$

By symmetry, $H_{\zeta^{2}}(-1,-1)=H_{\zeta^{2}}^{2}(-1)$; finishing the proof of (2.12.a).

Since $\left\{\xi_{m, n}\right\}$ has the strong commutation property and is strongly purely non-deterministic one can apply Theorem 2.11 to $\left\{\xi_{m, n}\right\}$, giving (2.12.b).

The proof of (2.12.c) is obvious.

\section{Analytical solution}

Our method to obtain an analytical expression for the prediction error $\left\|e^{4}(x)\right\|^{2}$ is based on the spectral representation for the covariance kernel of the innovation field $\left\{W_{m, n}^{1}(x)\right\}$ obtained by Korezlioglu and Loubaton [8]. According to Proposition II.2 and (IV.8) in [8] the covariance spectral measure of $\left\{W_{m, n}^{1}(x)\right\}$ has the properties

$$
d \nu_{W^{1}(x)}(u, v)=\frac{1}{2 \pi} d u d \rho_{x}^{1}(v)
$$

with 


$$
d \rho_{x}^{1}(v)=\int_{u=-\pi}^{\pi} d \nu_{W^{1}(x)}(u, v)
$$

and

$$
\frac{d \rho_{x}^{1}(v)}{d v}=\frac{1}{2 \pi} \exp \left\{\frac{1}{2 \pi} \int_{-\pi}^{\pi} \log f_{x}(u, v) d u\right\}
$$

as the absolutely continuous part of $d \rho_{x}^{1}$ with respect to the normalized Lebesgue measure $d v$ on $[-\pi, \pi)$. By symmetry, the same properties hold for $d \nu_{W^{2}(x)}$.

3.2. Remark. It clearly follows from (3.1.a) that the covariance spectral measure of the stationary sequence $\left\{W_{0, n}^{1}(x)\right\}_{n \in Z}$ is $d \rho_{x}^{1}$. Furthermore, by the well-known prediction theoretical results on stationary sequences only $d \rho_{x}^{1}(v) / d v$ is needed in calculating the prediction error of $\left\{W_{0, n}^{1}(x)\right\}$ needed in Proposition 2.4 (see e.g. [12], pp. 63-71). The spectral counterpart of (2.4) is well-known [2]. However, (2.4) combined with (3.1.c) gives a simple method to obtain

$$
\left\|e^{2}(x)\right\|^{2}=\exp \left\{\frac{1}{(2 \pi)^{2}} \int_{-\pi}^{\pi} \int_{-\pi}^{\pi} \log f_{x}(u, v) d u d v\right\} .
$$

The next example shows that $d \rho_{x}^{1}$ need not be absolutely continuous with respect to $d v$.

3.4. Example. Let $\left\{x_{m, n}\right\}$ be a bivariate and, respectively, $\left\{f_{m}\right\}_{m \in Z}$, a univariate white noise. Assume, in addition $f_{k} \perp x_{m, n}, k, m, n \in Z$. Define

$$
z_{m, n}=x_{m, n}+f_{m}, \quad(m, n) \in Z^{2}
$$

Then, $W_{m, n}^{1}(z)=z_{m, n},(m, n) \in Z^{2}$, i.e., $\left\{W_{0, n}^{1}(z)\right\}_{n \in Z}$, contains a deterministic component. Moreover, obviously

$$
d \nu_{z}(u, v)=d \nu_{W^{1}(x)}=\frac{1}{(2 \pi)^{2}} d u d v+\frac{1}{2 \pi} d u \otimes \delta_{0},
$$

where $\delta_{0}$ is the Dirac measure concentrated at the origin.

Our method to derive an analytical expression for the prediction error $\left\|e^{4}(x)\right\|^{2}$ is based on the spectral counterpart of the four-fold decomposition (2.10.a) obtained by Korezlioglu and Loubaton [8; Corollary III.13] and, under the weak commutation condition

$$
\operatorname{Proj}_{H_{x}^{1}(m)} \operatorname{Proj}_{H_{x}^{2}(n)}=\operatorname{Proj}_{H_{x}^{2}(n)} \operatorname{Proj}_{H_{x}^{1}(m)}, \quad m, n \in Z
$$


independently in [5; Theorem II.12]. According to Corollary III.13 [8], for any stationary random field $\left\{x_{m, n}\right\}$ one has $\xi_{m, n}(x) \neq 0$, if and only if

$$
\left\{\begin{array}{l}
\int_{-\pi}^{\pi} \log f_{x}(u, v) d u>-\infty \\
\int_{-\pi}^{\pi} \log f_{x}(u, v) d v>-\infty
\end{array}\right.
$$

and, under this condition,

$$
\begin{aligned}
& d \nu_{\xi(x)}=\frac{1}{(2 \pi)^{2}} \log f_{x}(u, v) d u d v \\
& d \zeta^{1}(x)=\frac{1}{2 \pi} \frac{d \nu_{x}(u, v)}{d u d \rho_{x}^{1, s}(v)} d u d \rho_{x}^{1, s}(v), \\
& d \zeta^{2}(x)=\frac{1}{2 \pi} \frac{d \nu_{x}(u, v)}{d \rho_{x}^{2, s}(u) d v} d \rho_{x}^{2, s}(u) d v
\end{aligned}
$$

where $d \rho_{x}^{1, s}(v)$ and $d \rho_{x}^{2, s}(u)$ are the singular parts with respect to the Lebesgue measure of $d \rho_{x}^{1}(v)$ and $d \rho_{x}^{2}(u)$, respectively. For brevity we state the spectral counterparts of Theorems 2.11 and 2.12 only under the assumption (3.6).

3.8. Remark. Let $\left\{x_{m, n}\right\}$ be a stationary random field. In what follows we use the notation

$$
\begin{aligned}
d^{4}\left(x^{a}\right) & =\frac{1}{2 \pi} \int_{-\pi}^{\pi} \exp \left\{\frac{1}{2 \pi} \int_{-\pi}^{\pi} \log f_{x}(u, v) d u\right\} d v \\
& +\frac{1}{2 \pi} \int_{-\pi}^{\pi} \exp \left\{\frac{1}{2 \pi} \int_{-\pi}^{\pi} \log f_{x}(u, v) d v\right\} d u \\
& -\exp \left\{\frac{1}{(2 \pi)^{2}} \int_{-\pi}^{\pi} \int_{-\pi}^{\pi} \log f_{x}(u, v) d u d v\right\} .
\end{aligned}
$$

3.9. Theorem. Let $\left\{x_{m, n}\right\}$ be a stationary random field having the strong commutation property. If $\left\{x_{m, n}\right\}$ is strongly purely non-deterministic, then

$$
d v_{x} \ll d u d v \quad \text { and (3.6) holds, }
$$

and

$$
\left\|e^{4}(x)\right\|^{2}=d^{4}\left(x^{a}\right) .
$$

3.10. Theorem. Let $\left\{x_{m, n}\right\}$ be a stationary random field having the strong commutation property. If (3.6) holds, then

$$
\begin{aligned}
\left\|e^{4}(x)\right\|^{2} & =\left\|e^{4}(\xi(x))\right\|^{2}+\left\|\zeta_{0,0}^{1}(x)-\zeta_{0,0}^{1}(x) / H_{\zeta^{1}(x)}^{1}(-1)\right\|^{2} \\
& +\left\|\zeta_{0,0}^{2}(x)-\zeta_{0,0}^{2}(x) / H_{\zeta^{2}(x)}^{2}(-1)\right\|^{2}
\end{aligned}
$$

with 


$$
\begin{aligned}
& \left\|e^{4}(\xi(x))\right\|^{2}=d^{4}\left(x^{a}\right) \\
& \left\|\zeta_{0,0}^{1}(x)-\zeta_{0,0}^{1}(x) / H_{\zeta^{1}(x)}^{1}(-1)\right\|^{2}= \\
& \int_{-\pi}^{\pi} \exp \left\{\frac{1}{2 \pi} \int_{-\pi}^{\pi} \log \left[\frac{d \nu_{x}(u, v)}{d u d \rho_{x}^{1, s}(v)}\right] d u\right\} d \rho_{x}^{1, s}(v),
\end{aligned}
$$

$$
\begin{aligned}
& \left\|\zeta_{0,0}^{2}(x)-\zeta_{0,0}^{2}(x) / H_{\zeta^{2}(x)}^{2}(-1)\right\|^{2}= \\
& \int_{-\pi}^{\pi} \exp \left\{\frac{1}{2 \pi} \int_{-\pi}^{\pi} \log \left[\frac{d \nu_{x}(u, v)}{d \rho_{x}^{2, s}(u) d v}\right] d v\right\} d \rho_{x}^{2, s}(u) .
\end{aligned}
$$

Before presenting proofs of Theorems 3.9 and 3.10 we continue Example 3.4.

3.11. Example. Let $\left\{z_{m, n}\right\}$ be defined according to (3.4). It is obvious that $\left\{z_{m, n}\right\}$ has the strong commutation property and $\xi_{m, n}(z)=x_{m, n}, \zeta_{m, n}^{1}(z)=f_{m}$, $\zeta_{m, n}^{2}(z)=0, \eta_{m, n}(z)=0,(m, n) \in Z^{2}$. Moreover,

$$
d \rho_{x}^{1}(v)=\int_{u=-\pi}^{\pi} d \nu_{W^{1}(x)}(u, v)=\frac{1}{2 \pi} d v+\delta_{0} .
$$

It is obvious, that

$$
\zeta_{m, n}^{1}(x) / H_{\zeta^{1}(x)}^{1}(m-1)=0 \quad \text { and } \quad \zeta_{m, n}^{1}(x)-\zeta_{m, n}^{1}(x) / H_{\zeta^{1}(x)}^{1}(m-1)=f_{m} .
$$

Proof of Theorem 3.9. Theorem III.12 together with Corollary III.13 in [8] imply that the properties (3.9.a) hold for any strongly purely non-deterministic stationary random field. The expression (3.9.b) then follows straightforwardly from (2.11.b) by using (3.1.c) and (3.3).

Proof of Theorem 3.10. The formula (3.10.a) is just a reformulation of (2.12.c). Since $\left\{\xi_{m, n}(x)\right\}$ has, by Theorem 2.1 [4], the strong commutation property (3.10.b) follows from (3.7.a) combined with Theorem 3.9.

By symmetry it is enough to justify (3.10.c) to finish the proof. It follows from Theorem II.1 [5] (cf. Proposition II.11 [7]) that

$$
d \nu_{W^{1}\left(\zeta^{1}(x)\right)}(u, v)=\frac{1}{2 \pi} \exp \left\{\frac{1}{2 \pi} \int_{-\pi}^{\pi} \log \left[\frac{d \nu_{x}(u, v)}{d u d \rho_{x}^{1, s}(v)}\right] d u\right\} \rho_{x}^{1, s}(v) .
$$

The formula (3.10.c) follows then immediately.

\section{References}

[1] Chiang Tse Pei: On the linear extrapolation of a continuous time homogeneous random field. - Theory Prob. Appl. 2, 1957, 58-89 (English translation of Teor. Veroyatnost. i Primenen. 2, 1957, 60-91). 
[2] Helson, H., and D. Lowdenslager: Prediction theory and Fourier series in several variables. - Acta Math. 99, 1958, 165-202.

[3] Helson, H., and D. Lowdenslager: Prediction theory and Fourier series in several variables II. - Acta Math. 106, 1961, 173-213.

[4] Kallianpur, G., and V. Mandrekar: Nondeterministic random fields and Wold and Halmos decompositions for commuting isometries. - Prediction theory and harmonic analysis. The Pesi Masani Volume, V. Mandrekar and H. Salehi (eds.). North-Holland, Amsterdam, 1983, 165-190.

[5] Kallianpur, G., A.G. Miamee, and H. Niemi: On the prediction theory of two parameter stationary random fields. - Technical Report 178, Center for Stochastic Processes. University of North Carolina, 1987.

[6] Kallianpur, G., A.G. Miamee, and H. Niemi: On the prediction theory of two parameter stationary random fields. - To appear.

[7] Korezlioglu, H., and P. Loubaton: Spectral factorization of wide sense stationary processes on $Z^{2}$. - J. Multivariate Anal. 19, 1986, 24-47.

[8] Korezlioglu, H., and P. Loubaton: Prediction and spectral decomposition of wide sense stationary processes on $Z^{2}$. - Spatial processes and spatial time series analysis. Proceedings of the 6th Franco-Belgian Meeting of Statisticians, November 1985, F. Droesbeke (ed.). Publications des Facultes universitaires Saint-Louis, Bruxelles, 1987, 127-164.

[9] Makagon,A., and H. Salehi: Stationary fields with positive angle. - J. Multivariate Anal. 22, 1987, 106-125.

[10] Miamee, A.G., and H. Niemi: On the angle for stationary random fields. - Technical Report 92, Center for Stochastic Processes, University of North Carolina, 1985.

[11] Rosenblatt, M.: Stationary sequences and random fields. - Birkhäuser, Boston, 1985.

[12] Rozanov, Yu.: Stationary random processes. - Holden-Day, San Francisco, 1967.

[13] Soltani, A.R.: Extrapolation and moving average representation for stationary random fields and Beurling's theorem. - Ann. Probab. 12, 1984, 120-132.

University of Helsinki

Department of Statistics

Aleksanterinkatu 7

SF-00100 Helsinki

Finland 